\title{
Polymorphic Eruption of Pregnancy Presented with Targetoid Lesions: A Report of Two Cases
}

\author{
Wararat Sirikudta Narumol Silpa-archa \\ Department of Dermatology, Faculty of Medicine Siriraj Hospital, Mahidol University, \\ Bangkok, Thailand
}

\section{Key Words}

Polymorphic eruption of pregnancy - Pruritic urticarial papules and plaques of pregnancy . Targetoid lesion

\begin{abstract}
Background: Skin lesions in pregnant women could be caused by physiologic or pathologic changes. Polymorphic eruption of pregnancy (PEP), which manifests as various types of skin lesions, is the most common pregnancy dermatosis. Thus, PEP could mimic other skin diseases related to unfavorable maternal and fetal outcomes. Main Observations: Two PEP patients with targetoid lesions are presented here. One of them was a primigravida, whereas the other was a secundigravida. Both patients had singleton pregnancies and skin rash which started during the third trimester. The lesions began on the abdomen and then spread to the trunk and extremities. The face, palms, soles, and mucosa were not affected. Pruritus was observed but no other systemic symptoms were reported. Both patients delivered healthy, term infants without complications. Conclusion: Targetoid lesions in PEP are an uncommon presentation, and the differential diagnosis of PEP along with other dermatoses should be considered. However, the prognosis for this type of PEP is not different from that for classic PEP.
\end{abstract}

\section{Introduction}

Pregnant women undergo many physiologic changes in order to accommodate the fetus. Some skin changes during pregnancy are a physiologic adaptation, for example, generalized hirsutism, linea nigra, secondary areola, spider angioma, and melasma [1]. During pregnancy, various dermatoses can occur, some of which are related to adverse fetal outcome while 
others are not [2]. Polymorphic eruption of pregnancy (PEP) is the most common pregnancy dermatosis. This condition usually occurs in late pregnancy of primigravidas, and the patients can present with polymorphic lesions, urticarial papules and plaques, eczematous lesions, vesicles, nonurticarial erythema, or, less likely, targetoid lesions [3]. Despite these eruptions, the fetus has an excellent prognosis [1]. Due to diverse clinical manifestations of PEP, some of the skin lesions can be confused with many other dermatoses. Herein, we report 2 cases of PEP which presented with targetoid pruritic skin lesions, and a review of the literature has also been performed.

\section{Case 1}

A 30-year-old Thai female $\mathrm{G}_{2} \mathrm{P}_{0} \mathrm{~A}_{1}$ at $37^{+1}$ weeks of gestation presented with a 1-week history of pruritic erythematous papules and plaques starting on the lower abdomen. The rash spread to all extremities and the trunk; however, mucosa, palms, and soles were spared. Neither abnormal symptoms nor signs of systemic involvement or fetal distress were detected.

She had been receiving regular antenatal care since she was 8 weeks pregnant. Her medications were 1 ferli- 6 tab per day and $1 \mathrm{~g}$ calcium carbonate per day, which she had been taking since an antenatal care visit. She had undergone an abortion during the eighth week of pregnancy 5 years earlier. There was no history of a similar skin condition. She denied any previous herpes simplex infection. Physical examination revealed generalized discrete and confluent erythematous papules and plaques with central dusky red areas (targetoid lesions) on the trunk and extremities (fig. 1). Some tiny pustules and vesicles were noted on both arms and the chest wall. Conjunctiva and oral and genital mucosa were unremarkable.

A Tzanck smear from the vesicle showed the presence of polymorphonuclear cells but the absence of multinucleated giant cells. Gram staining of the pustules showed no organisms. Direct immunofluorescent study was negative for herpes simplex virus antigen. Additionally, a skin biopsy taken from the targetoid lesions on the left forearm illustrated focal parakeratosis with scale crust formation, irregular acanthosis and focal spongiosis of the epidermis, superficial perivascular infiltration of lymphocytes, and eosinophils with red blood cell extravasation. Direct and indirect immunofluorescent studies (HG factor) were all negative. Due to the diversity of skin manifestations, excluding the cause of the targetoid lesions and the negative result of laboratory investigations, PEP was diagnosed.

Systemic corticosteroids were prescribed at the patient's first visit because pemphigoid gestationis could not be excluded. However, after the diagnosis of PEP was made, only topical corticosteroids and oral antihistamines were prescribed; the patient had a good clinical response. She delivered a healthy, term, male infant.

\section{Case 2}

A 26-year-old Thai female $\mathrm{G}_{1} \mathrm{P}_{0}$ at $39^{+4}$ weeks of gestation presented with a 1-week history of severely pruritic erythematous papules and plaques beginning on the abdomen and subsequently involving all extremities. Neither systemic symptoms nor fetal distress was observed. She had been receiving standard antenatal care since she was 13 weeks pregnant. Her medication was only ferli- 6 and calcium carbonate. Physical examination showed multiple, discrete, ill-defined, erythematous papules and confluent plaques with 
Sirikudta et al.: Polymorphic Eruption of Pregnancy Presented with Targetoid Lesions: A Report of Two Cases

central dusky red areas (targetoid lesions) on the abdomen, back, and all extremities (fig. 2). Palms, soles, and mucosa were not affected. An indirect immunofluorescent study was done to exclude pemphigoid gestationis, and the result was negative. Systemic corticosteroids were initially given. Then, topical corticosteroids together with oral antihistamines were prescribed instead. Her symptoms improved over time and she delivered a healthy, term, female infant.

\section{Discussion}

PEP is the most common specific skin eruption in pregnancy. Due to the variety of its skin lesions, there are many terminologies for this condition, such as toxic erythema of pregnancy, Bourne's toxemic rash of pregnancy, Nurse's late onset prurigo of pregnancy, and pruritic urticarial papules and plaques of pregnancy [1]. The classic clinical presentation of PEP constitutes intensely pruritic urticarial papules and plaques starting within and/or adjacent to striae, sparing the periumbilical area. Later on, the lesions can spread to nonabdominal sites, such as the buttocks and thighs; however, the face, palms, soles, and mucosa are usually not involved [3]. Approximately half of the patients later develop polymorphic skin lesions, such as urticarial papules and plaque (49\%), eczematous lesions $(22 \%)$, vesicles $(17 \%)$, nonurticarial erythema (6\%), and targetoid lesions (6\%). Systemic symptoms are usually absent $[4,5]$.

PEP is related to male fetuses and multiple gestation and usually occurs in the third trimester of pregnancy of primigravidas $[3,4,6]$. However, onset in the immediate postpartum period has also been reported [7]; whether it is associated with maternal or fetal weight gain or maternal atopic background is still debatable $[4,6,8]$.

Etiologies of PEP are proposed by many authors [6, 9-13]. First, the exposure of collagen bundle antigens resulting from abdominal overdistension during late pregnancy could be the cause. This could also explain the clinical manifestation of the skin rash which is typically predominant on abdominal striae, and the fact that most patients experience disease onset in the third trimester. Second, because fetal DNA has been detected in skin lesions of PEP patients, this could indicate a maternal allergic reaction to fetal DNA [9]. Lastly, hormonal changes have been reported to be related to PEP; however, this is still debatable [10-12]. Although the first patient in this report is multigravida, her first pregnancy was terminated during the eighth week of pregnancy, when the abdomen had not been distended. Thus, the abdominal overdistension theory supports this multigravida clinical setting.

Regarding the differential diagnosis, both of our patients were in the third trimester of pregnancy and presented with striking targetoid lesions on the trunk and extremities, combined with some vesicles and pustules; erythema multiforme, PEP and pemphigoid gestationis should be of concern. In erythema multiforme, a history of previous herpes simplex infection and drug allergy should be considered. Pemphigoid gestationis is a rare autoimmune vesiculobullous disease with autoantibodies against basement membranes; it presents with pruritic erythematous papule, plaque, and blisters [1, 2]. However, there is a report of targetoid lesions in pemphigoid gestationis [14]. Immunofluorescent studies should be done to guide the diagnosis [1].

Aronson et al. [5] reported 57 patients with PEP and revealed various types of skin lesions, of which targetoid lesions represented 5.3\%. In addition, Rudoph et al. [4] reported that $11(6 \%)$ out of 181 PEP patients had targetoid lesions. Specific characteristics of patients with targetoid lesions are not described in these 2 studies. Ghazeeri et al. [8] 
Sirikudta et al.: Polymorphic Eruption of Pregnancy Presented with Targetoid Lesions: A Report of Two Cases

demonstrated that only 1 (5.6\%) out of 18 PEP patients had targetoid lesions. This patient was a 32-year-old primigravida and had a singleton pregnancy. She developed urticarial papules, plaques, and targetoid lesions on the abdomen, thighs, and legs at 26 weeks of gestation. She had a vaginal delivery without complications. As in previous studies, the prevalence of targetoid lesions in PEP was quite similar and no fetal complications were reported [8].

A histopathological examination should be performed in order to rule out other pregnancy dermatoses such as pemphigoid gestationis; histological changes in PEP are nonspecific. Early PEP lesions are characterized by epidermal and upper dermal edema as well as a superficial perivascular infiltrate predominantly composed of lymphohistiocytes with occasional eosinophils. Focal spongiosis can be present in vesicular lesions. In addition, focal parakeratosis and acanthosis can be detected in resolving lesions [3]. Both direct and indirect immunofluorescent studies are generally negative in PEP patients. However, Aronson et al. [5] reported that 15 of 57 patients had deposition of IgM, C3 or IgA at the dermo-epidermal junction or in the blood vessels. The first patient presented herein had no specific histological findings and had a negative result from immunofluorescent studies, which supported the diagnosis of PEP.

PEP is a benign and self-limiting condition. The duration of eruptions is approximately 4-6 weeks [1, 3]. Symptomatic treatment with topical corticosteroids and oral firstgeneration antihistamines is safe and usually enough to control the eruptions. Systemic corticosteroids could be prescribed in patients with extensive disease [2, 8]. Furthermore, both the affected pregnant women and their fetuses have a favorable prognosis. Recurrence in any subsequent pregnancies is uncommon $[3,15]$. Cutaneous lesions in our 2 patients gradually improved, and no adverse fetal outcome was detected.

In conclusion, targetoid lesions in PEP are an uncommon presentation, and the differential diagnosis of PEP along with other dermatoses should be considered. Nonetheless, the prognosis is not different to that of classic PEP.

\section{Disclosure Statement}

The authors declare no conflict of interest.

\section{References}

1 Roth MM: Pregnancy dermatoses: diagnosis, management, and controversies. Am J Clin Dermatol 2011;12:25-41.

-2 Kroumpouzos G, Cohen LM: Specific dermatoses of pregnancy: an evidence-based systematic review. Am J Obstet Gynecol 2003;188:1083-1092.

3 Ahmadi S, Powell FC: Pruritic urticarial papules and plaques of pregnancy: current status. Australas J Dermatol 2005;46:53-58, quiz 59.

4 Rudolph CM, Al-Fares S, Vaughan-Jones SA, Mullegger RR, Kerl H, Black MM: Polymorphic eruption of pregnancy: clinicopathology and potential trigger factors in 181 patients. Br J Dermatol 2006;154:54-60.

-5 Aronson IK, Bond S, Fiedler VC, Vomvouras S, Gruber D, Ruiz C: Pruritic urticarial papules and plaques of pregnancy: clinical and immunopathologic observations in 57 patients. J Am Acad Dermatol 1998;39:933939.

6 Cohen LM, Capeless EL, Krusinski PA, Maloney ME: Pruritic urticarial papules and plaques of pregnancy and its relationship to maternal-fetal weight gain and twin pregnancy. Arch Dermatol 1989;125:1534-1536.

7 Buccolo LS, Viera AJ: Pruritic urticarial papules and plaques of pregnancy presenting in the postpartum period: a case report. J Reprod Med 2005;50:61-63.

8 Ghazeeri G, Kibbi AG, Abbas 0: Pruritic urticarial papules and plaques of pregnancy: epidemiological, clinical, and histopathological study of 18 cases from Lebanon. Int J Dermatol 2012;51:1047-1053. 
-9 Aractingi S, Berkane N, Bertheau P, Le Goue C, Dausset J, Uzan S, Carosella ED: Fetal DNA in skin of polymorphic eruptions of pregnancy. Lancet 1998;352:1898-1901.

10 Campbell DM: Maternal adaptation in twin pregnancy. Semin Perinatol 1986;10:14-18.

11 Im S, Lee ES, Kim W, Song J, Kim J, Lee M, Kang WH: Expression of progesterone receptor in human keratinocytes. J Korean Med Sci 2000;15:647-654.

12 Vaughan Jones SA, Hern S, Nelson-Piercy C, Seed PT, Black MM: A prospective study of 200 women with dermatoses of pregnancy correlating clinical findings with hormonal and immunopathological profiles. Br J Dermatol 1999;141:71-81.

13 Garcia-Gonzalez E, Ahued-Ahued R, Arroyo E, Montes-De-Oca D, Granados J: Immunology of the cutaneous disorders of pregnancy. Int J Dermatol 1999;38:721-729.

$\checkmark 14$ Ogilvie P, Trautmann A, Dummer W, Rose C, Brocker EB, Zillikens D: Pemphigoid gestationis without blisters (in German). Hautarzt 2000;51:25-30.

15 Matz H, Orion E, Wolf R: Pruritic urticarial papules and plaques of pregnancy: polymorphic eruption of pregnancy (PUPPP). Clin Dermatol 2006;24:105-108.
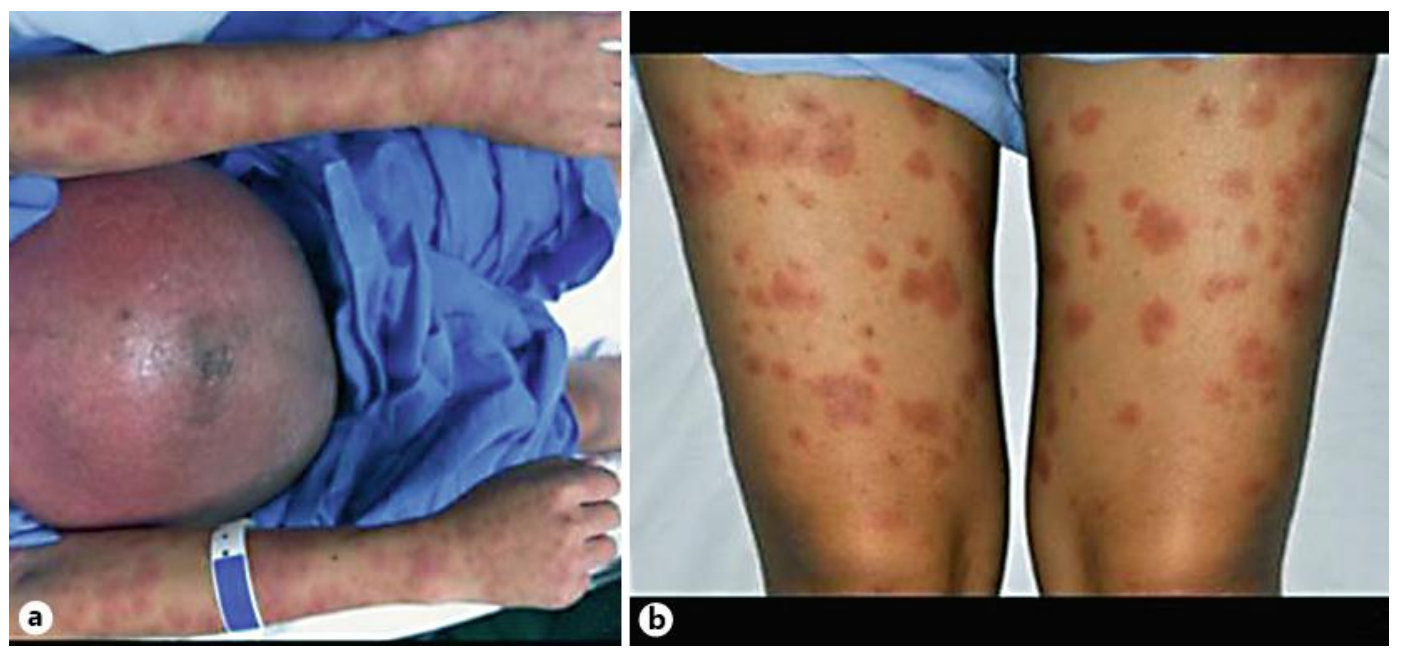

Fig. 1. A $\mathrm{G}_{2} \mathrm{P}_{0} \mathrm{~A}_{1}$ at $37^{+1}$ weeks of gestation presented with generalized discrete and confluent erythematous papules and plaques with central dusky red areas (targetoid lesions) on the abdomen and upper extremities (a) and the lower extremities (b). 
Sirikudta et al.: Polymorphic Eruption of Pregnancy Presented with Targetoid Lesions: A Report of Two Cases

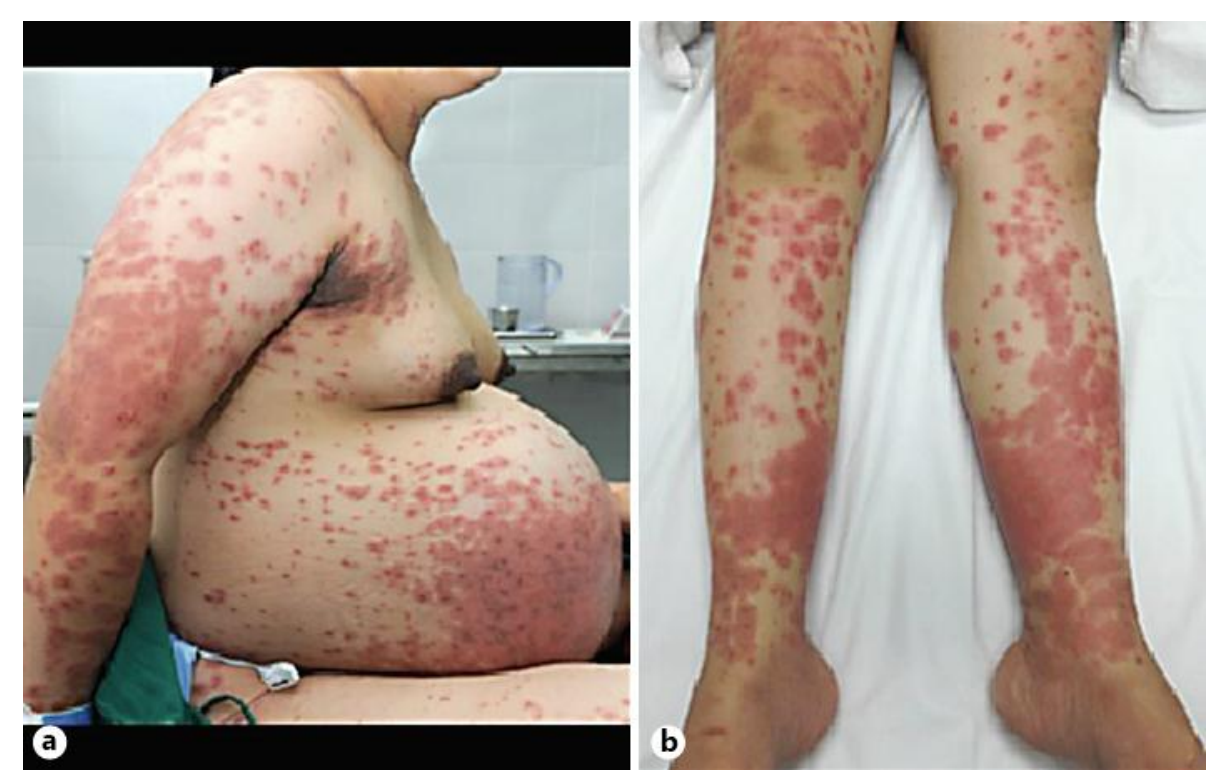

Fig. 2. $A \mathrm{G}_{1} \mathrm{P}_{0}$ at $39^{+4}$ weeks of gestation developed discrete, ill-defined, erythematous papules and confluent plaques with central dusky red areas (targetoid lesions) on the abdomen, back, and upper extremities (a) as well as the lower extremities (b). 